\title{
The effect of carbamylation on the functionality of high-density
} lipoprotein

\author{
Michael Holzer*1, Martin Gauster ${ }^{2}$, Ruth Birner-Grünberger ${ }^{3}$ and \\ Gunther Marsche ${ }^{1}$
}

Address: ${ }^{1}$ Institute of Experimental and Clinical Pharmacology, Medical University of Graz, 8010 Graz, Austria, ${ }^{2}$ Institute for Cell Biology, Histology and Embryology, Medical University of Graz, 8010 Graz, Austria and ${ }^{3}$ Core Facility Mass Spectrometry, Medical University of Graz, 8010 Graz, Austria

Email: Michael Holzer* - michael.holzer@medunigraz.at

* Corresponding author

\author{
from I5th Scientific Symposium of the Austrian Pharmacological Society (APHAR) Joint meeting with the Hungarian Society of Experimental and Clinical \\ Pharmacology (MFT) and the Slovenian Pharmacological Society (SDF) \\ Graz, Austria. 19-21 November 2009 \\ Published: 12 November 2009 \\ BMC Pharmacology 2009, 9(Suppl 2):A6 doi:10.1186/1471-2210-9-S2-A6
}

This abstract is available from: http://www.biomedcentral.com//47I-22/0/9/S2/A6

(c) 2009 Holzer et al; licensee BioMed Central Ltd.

\section{Background}

Increasing interest has focused on the relative functionality of high-density lipoprotein (HDL), highlighted by observations that cardiovascular events can occur even in the presence of high levels of HDL cholesterol. Myeloperoxidase (MPO), a heme protein abundant in leucocytes, colocalizes with HDL in the human artery wall and has emerged as a potential participant in multiple phases of the atherosclerotic process. Recently, the $\mathrm{MPO} / \mathrm{H}_{2} \mathrm{O}_{2} /$ $\mathrm{SCN}-$ system has been demonstrated as a dominant pathway to promote protein carbamylation within atherosclerotic plaques. Therefore, we determined whether HDL is carbamylated in the human artery wall.

\section{Methods and results}

Immunohistochemical studies confirmed colocalization of carbamylated epitopes with apoA-I and macrophages in human atherosclerotic lesions. We performed shotgun proteomic analysis of in vitro carbamylated HDL to identify specific carbamylation sites of apoA-I. We could identify apoA-I-associated lysine residues in the $\alpha$-helical lipid binding domains that are specifically carbamylated, indicating that carbamylation of apoA-I affects the functional integrity of HDL. In line with this observation, we observed that carbamylation of HDL (i) leads to "nonproductive" binding to the HDL receptor (SR-BI), (ii) decreased SR-BI-mediated cholesterol efflux, and (iii) reduced HDL mediated anti-inflammatory activity.

\section{Conclusion}

Taken together, our data provide strong evidence that carbamylation renders HDL dysfunctional and proinflammatory. 